\title{
A Higher-Order Finite Difference Scheme for Singularly Perturbed Parabolic Problem
}

\author{
Shifang Tian (D), Xiaowei Liu $(\mathbb{D}$, and Ran An \\ School of Mathematics and Statistics, Qilu University of Technology (Shandong Academy of Sciences), Jinan 250353, China \\ Correspondence should be addressed to Xiaowei Liu; xwliuvivi@hotmail.com
}

Received 3 April 2021; Accepted 22 July 2021; Published 20 August 2021

Academic Editor: Hui Wang

Copyright (C) 2021 Shifang Tian et al. This is an open access article distributed under the Creative Commons Attribution License, which permits unrestricted use, distribution, and reproduction in any medium, provided the original work is properly cited.

In this paper, we deal with a singularly perturbed parabolic convection-diffusion problem. Shishkin mesh and a hybrid third-order finite difference scheme are adopted for the spatial discretization. Uniform mesh and the backward Euler scheme are used for the temporal discretization. Furthermore, a preconditioning approach is also used to ensure uniform convergence. Numerical experiments show that the method is first-order accuracy in time and almost third-order accuracy in space.

\section{Introduction}

We consider the singularly perturbed parabolic problem posed on the domain $G=\Omega_{x} \times \Omega_{t}=(0,1) \times(0, T]$ as follows:

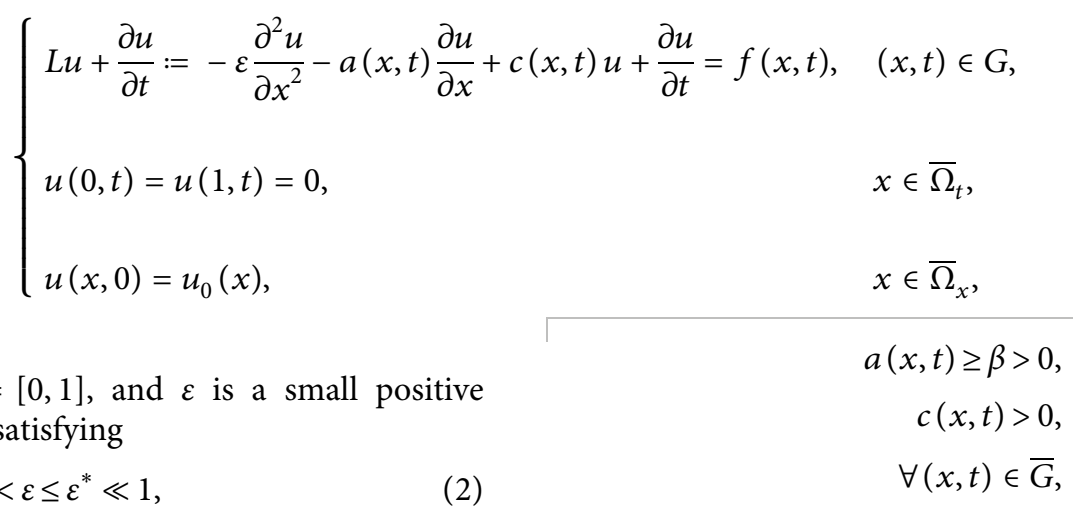

where $\beta$ is a positive constant.

With these conditions, there exists a unique with a positive constant $\varepsilon^{*}$, and $u_{0}(x)$ is the initial value when $t=0$. We assume that the functions $a(x, t), c(x, t)$, and $f(x, t)$ are sufficiently smooth, and that $a(x, t)$ and $c(x, t)$ satisfy 
function $u$ to its fourth partial derivative for spatial variable $u_{x x x x}$ are continuous, and solutions from its original function $u$ to its second partial derivative for temporal variable $u_{t t}$ are also continuous.

Singular perturbation problems play an important role in many areas, such as astronomy, mechanics, and fluid dynamics. It also has a broad background and important applications in control systems with different time scales [2-4]. It is especially important to find a uniform and effective approximate solution when the exact solution cannot be obtained. There are many methods for solving singular perturbation problems. Recent convergence analysis of the finite element method is referred to [5-14]. Except the finite element method, the finite difference method is the most widely used one at present. Nowadays, more and more people begin to study higher-order finite difference schemes for solving singular perturbation problems. In 1988, Vulanović in [15] proposed a third-order hybrid finite difference scheme and showed numerical results on the Shishkin mesh. Afterwards, Vulanovic and Nhan in [16] improved on what had already been done and proposed a new uniformly convergent numerical scheme. Both the methods proposed in $[15,17]$ have been analyzed on a piecewise-uniform Shishkin mesh and were proved to be almost third-order accuracy in space. Comparing the previous scheme, the difference is that when $\varepsilon$ is large enough, the accuracy of the new scheme is better than the that of the previous scheme. However, when $\varepsilon$ is small enough, there is no difference between these two methods.

In this paper, our primary aim is to propose and analyze a higher-order hybrid finite difference scheme for problem (1). This is accomplished by discretizing the domain $\Omega_{x}$ using the Shishkin mesh and by considering the uniform mesh in the temporal direction. In order to obtain the fully discrete scheme, we adopt the two-stage discretization process. The first stage consists of discretizing the time derivative with the backward difference scheme on the uniform mesh. In the second stage, discretize in the spatial direction by utilizing a hybrid finite difference scheme on the Shishkin mesh.

The ultimate goal of numerical methods for problem (1) is to obtain a series of discrete solutions so as to achieve a numerical approximation of the continuous solution. Such that its error converges to 0 uniformly as $N \longrightarrow+\infty$, where $N$ is the number of discretization on the spatial mesh. Apart from this, in numerical experiments, we also need to illustrate that the proposed scheme is almost third-order accurate in space.

The rest of the paper is as follows. In Section 2, we define the meshes for temporal and spatial discretization and introduce some special difference operators. In Section 3, we define some difference operators and the final finite difference scheme. In Section 4, we give the linear equations needed to solve the problem and get the coefficient matrix and the right end term. In Section 5, we preprocess the coefficient matrix. In Section 6, we give the pseudo code to solve the problem. In Section 7, we give the results of numerical experiments. In Section 8 , some final conclusions are given.

\section{The Mesh}

Here, in this section, we describe the uniform mesh for the temporal discretization of the domain $\Omega_{t}$ and the Shishkin mesh for the spatial discretization of the domain $\Omega_{x}$.

We will often use the assumption that

$$
\varepsilon \leq C_{*} N^{-1},
$$

where $C_{*}$ is a sufficiently small positive constant which is independent of both $\varepsilon$ and $N$. All constants, independent of $\varepsilon$ and $N$, are denoted generically by $C$.

2.1. The Uniform Mesh. For the time domain $[0, T]$, we use a uniform mesh with time step $\Delta t$, such that

$$
\Omega_{t}^{M}=\left\{t_{n}=n \Delta t \quad n=0, \ldots, M \Delta t=\frac{T}{M}\right\},
$$

where $M$ is the number of mesh points in the $t$-direction on the interval $[0, T]$.

2.2. Shishkin Mesh. Since problem (1) has a boundary layer along the side $x=1$, the mesh should be condensing in the neighborhood of $x=1$. Define

$$
\sigma=\min \left\{\frac{1}{2}, \frac{\alpha \varepsilon}{\beta} \ln N\right\},
$$

with $\alpha \geq 4$ (the proof of range of $\alpha$ was given in [16]). To define the piecewise-uniform mesh, we divide the domain $[0,1]$ into two subdomains, such that $[0,1]=[0,1-\sigma] \cup[1-\sigma, 1]$ and then divide each of the subdomains into $(N / 2)$ equal intervals. Set

$$
\begin{cases}h:=h_{i}=\frac{(1-\sigma)}{N}, & \text { for } i=0,1,2, \ldots,(N / 2), \\ H:=h_{i}=\frac{\sigma}{N}, & \text { for } i=(N / 2)+1, \ldots, N\end{cases}
$$

Now, we denote the spatial grids by

$$
\Omega_{x}^{N}=\left\{0=x_{0}, x_{1}, \ldots, x_{N / 2}=1-\sigma, \ldots, x_{N}=1\right\},
$$

where

$$
x_{i}= \begin{cases}i h, & \text { for } i=0,1,2, \ldots,(N / 2), \\ 1-\sigma+\left(i-\frac{N}{2}\right) H, & \text { for } i=(N / 2)+1, \ldots, N,\end{cases}
$$

and $N \geq 4$ be a positive even integer. Here, the transition point $1-\sigma$ separates the coarse and fine portions of the mesh.

Moreover, define

$$
x_{i+z}= \begin{cases}x_{i}+z h_{i+1}, & \text { if } z \in[0,1), \\ x_{i}+z h_{i}, & \text { if } z \in(-1,0],\end{cases}
$$

where $z$ is some fixed constant. 


\section{Discretization}

In this section, we will give different difference operators corresponding to different points on the Shishkin mesh and combine these difference operators to form the final numerical scheme.
Firstly, we denote by $U_{i}^{j}$ the approximation of $u$ at point $\left(x_{i}, t_{j}\right)$ and set $f_{i+z}^{j}=f\left(x_{i+z}, t_{j}\right)$. Then, we use $D_{x, z}^{(0)} U_{i}^{j}$, $D_{\chi, z}^{(1)} U_{i}^{j}$, and $D_{\chi, z}^{(2)} U_{i}^{j}$ as the approximations of $u\left(x_{i+z}, t_{j}\right)$, $u_{x}\left(x_{i+z}, t_{j}\right)$, and $u_{x x}\left(x_{i+z}, t_{j}\right)$, respectively. They are defined by the following equation [16]:

$$
\left\{\begin{array}{l}
D_{\chi, z}^{(0)} U_{i}^{j}=\frac{1}{2}\left[z(z-1) U_{i-1}^{j}+2\left(1-z^{2}\right) U_{i}^{j}+z(z+1) U_{i+1}^{j}\right] \\
D_{\chi, z}^{(1)} U_{i}^{j}=\frac{1}{6 \chi}\left[\left(-3 z^{2}+6 z-2\right) U_{i-1}^{j}+3\left(3 z^{2}-4 z-1\right) U_{i}^{j}+3\left(-3 z^{2}+2 z+2\right) U_{i+1}^{j}+\left(3 z^{2}-1\right) U_{i+2}^{j}\right] \\
D_{\chi, z}^{(2)} U_{i}^{j}=\frac{1}{\chi^{2}}\left[(1-z) U_{i-1}^{j}+(3 z-2) U_{i}^{j}+(1-3 z) U_{i+1}^{j}+z U_{i+2}^{j}\right]
\end{array}\right.
$$

where $\chi$ is the step size of a uniform mesh and $z$ is a constant satisfying $z \in(-1,1)$. We set $e_{i+z}^{(n)}$ as the truncation error between the numerical solution and the exact solution.

$$
e_{i+z}^{(n)}=D_{\chi, z}^{(n)} U_{i}^{j}-u\left(x_{i+z}, t_{j}\right), \quad n=0,1,2 .
$$

Firstly, $e_{i+z}^{(0)}=D_{\chi, z}^{(0)} U_{i}^{j}-u\left(x_{i+z}, t_{j}\right)$.

Lemma 1. Suppose that

$$
\left|\frac{\partial^{m}}{\partial x^{m}} u(x, t)\right| \leq C, \quad(x, t) \in \bar{\Omega}_{x} \times \bar{\Omega}_{t}, m \geq 0 .
$$

The truncation error associated to $e_{i+z}^{(0)}$ satisfies

$$
\left\|e_{i+z}^{0}\right\|_{\infty} \leq C \chi^{3},
$$

where $\chi$ is the step size.

Proof. We substitute $u\left(x_{i-1}, t_{j}\right), u\left(x_{i}, t_{j}\right)$, and $u\left(x_{i+1}, t_{j}\right)$ for $U_{i-1}^{j}, U_{i}^{j}$, and $U_{i+1}^{j}$ in operator $D_{\chi, z}^{(0)} U_{i}^{j}$ and apply the Taylor expansion to obtain

$$
\begin{aligned}
e_{i+z}^{(0)}= & \frac{1}{2}\left[z(z-1)\left(u\left(x_{i}, t_{j}\right)-\chi u^{\prime}\left(x_{i}, t_{j}\right)+\frac{1}{2} \chi^{2} u^{\prime \prime}\left(x_{i}, t_{j}\right)-\frac{1}{6} \chi^{3} u^{3}\left(x_{i}, t_{j}\right)+\cdots\right)+2\left(1-z^{2}\right) u\left(x_{i}, t_{j}\right)\right. \\
& \left.+z(z+1)\left(u\left(x_{i}, t_{j}\right)-\chi u^{\prime}\left(x_{i}, t_{j}\right)+\frac{1}{2} \chi^{2} u^{\prime \prime}\left(x_{i}, t_{j}\right)-\frac{1}{6} \chi^{3} u^{3}\left(x_{i}, t_{j}\right)+\cdots\right)\right]-\left(u\left(x_{i}, t_{j}\right)-\chi u^{\prime}\left(x_{i}, t_{j}\right)+\frac{1}{2} \chi^{2} u^{\prime \prime}\left(x_{i}, t_{j}\right)\right. \\
& \left.-\frac{1}{6} \chi^{3} u^{3}\left(x_{i}, t_{j}\right)+\cdots\right)=\frac{1}{6} z \chi^{3} u^{3}\left(x_{i}, t_{j}\right)-\frac{1}{6} z^{3} \chi^{3} u^{3}\left(x_{i}, t_{j}\right)+O \chi^{4} .
\end{aligned}
$$

Thus,

$$
\left\|e_{i+z}^{(0)}\right\|_{\infty} \leq C \chi^{3} .
$$

Secondly, $e_{i+z}^{(1)}=D_{\chi, z}^{(1)} U_{i}^{j}-u\left(x_{i+z}, t_{j}\right)$.

Lemma 2. Suppose that

$$
\left|\frac{\partial^{m}}{\partial x^{m}} u(x, t)\right| \leq C, \quad(x, t) \in \bar{\Omega}_{x} \times \bar{\Omega}_{t}, m \geq 0 .
$$

The truncation error associated to $e_{i+z}^{(1)}$ satisfies

$$
\left\|e_{i+z}^{(1)}\right\|_{\infty} \leq C \chi^{3},
$$

where $\chi$ is the step size.

Proof. Similar to above, we substitute $u\left(x_{i-1}, t_{j}\right), u\left(x_{i}, t_{j}\right)$, $u\left(x_{i+1}, t_{j}\right)$, and $u\left(x_{i+2}, t_{j}\right)$ for $U_{i-1}^{j}, U_{i}^{j}, U_{i+1}^{j}$, and $U_{i+2}^{j}$ in operator $D_{\chi, z}^{(1)} U_{i}^{j}$ and again apply the Taylor expansion to obtain 


$$
\begin{aligned}
e_{i+z}^{(1)}= & \frac{1}{6 \chi}\left[\left(-3 z^{2}+6 z-2\right)\left(u\left(x_{i}, t_{j}\right)-\chi u^{\prime}\left(x_{i}, t_{j}\right)+\frac{1}{2} \chi^{2} u^{\prime \prime}\left(x_{i}, t_{j}\right)-\frac{1}{6} \chi^{3} u^{3}\left(x_{i}, t_{j}\right)+\cdots\right)\right. \\
& +3\left(3 z^{2}-4 z-1\right) u\left(x_{i}, t_{j}\right)+3\left(-3 z^{2}+2 z+2\right)\left(u\left(x_{x}, t_{j}\right)-\chi u^{\prime}\left(x_{i}, t_{j}\right)+\frac{1}{2} \chi^{2} u^{\prime \prime}\left(x_{i}, t_{j}\right)-\frac{1}{6} \chi^{3} u^{3}\left(x_{i}, t_{j}\right)+\cdots\right) \\
& \left.+\left(3 z^{2}-1\right)\left(u\left(x_{x}, t_{j}\right)-\chi u^{\prime}\left(x_{i}, t_{j}\right)+\frac{1}{2}(2 \chi)^{2} u^{\prime \prime}\left(x_{i}, t_{j}\right)-\frac{1}{6}(2 \chi)^{3} u^{3}\left(x_{i}, t_{j}\right)+\cdots\right)\right] \\
& -\left(u\left(x_{i}, t_{j}\right)-\chi z u^{\prime}\left(x_{i}, t_{j}\right)+\frac{1}{2}(\chi z)^{2} u^{\prime \prime}\left(x_{i}, t_{j}\right)-\frac{1}{6}(\chi z)^{3} u^{3}\left(x_{i}, t_{j}\right)+\cdots\right) \\
= & \frac{1}{6} z \chi^{3} u^{3}\left(x_{i}, t_{j}\right)-\frac{1}{6} z^{3} \chi^{3} u^{4}\left(x_{i}, t_{j}\right)+O \chi^{4}
\end{aligned}
$$

Thus,

$$
\left\|e_{i+z}^{(1)}\right\|_{\infty} \leq C \chi^{3}
$$

In conclusion, both $D_{\chi, z}^{(0)} U_{i}^{j}$ and $D_{\chi, z}^{(1)} U_{i}^{j}$ are third-order accurate with respect to the spatial variable $x$ for any value of $z$; if $z=(1 / \sqrt{3}), D_{\chi, z}^{(1)} U_{i}^{j}$ is transformed into the classical three-point scheme. And in the same way, operator $D^{\prime \prime} U_{i}^{j}$ (27), $\bar{D}^{(0)} U_{N / 2}^{j}$ (29), $\widehat{D}^{\prime \prime} U_{N / 2}^{j}$ (30), and time difference operator $D_{t}^{-} U_{i}^{j}$ can all be proven.

Moreover, $e_{i+z}^{(2)}=D_{\chi, z}^{(2)} U_{i}^{j}-u\left(x_{i+z}, t_{j}\right)$.

Lemma 3. Assume that

$$
\left|\frac{\partial^{m}}{\partial x^{m}} u(x, t)\right| \leq C, \quad(x, t) \in \bar{\Omega}_{x} \times \bar{\Omega}_{t}, m \geq 0 .
$$

The truncation error associated to $e_{i+z}^{(2)}$ satisfies that if $z=((3-\sqrt{15}) / 6)$,

$$
\left\|e_{i+z}^{(2)}\right\|_{\infty} \leq C \chi^{3}
$$

else

$$
\left\|e_{i+z}^{(2)}\right\|_{\infty} \leq C \chi^{2}
$$

where $\chi$ is the step size.

Proof. Once more, substituting $u\left(x_{i-1}, t_{j}\right), u\left(x_{i}, t_{j}\right)$, $u\left(x_{i+1}, t_{j}\right)$, and $u\left(x_{i+2}, t_{j}\right)$ for $U_{i-1}^{j}, U_{i}^{j}, U_{i+1}^{j}$, and $U_{i+2}^{j}$ in operator $D_{\chi, z}^{(2)} U_{i}^{j}$ and applying the Taylor expansion results in

$$
\begin{aligned}
e_{i+z}^{(2)}= & \frac{1}{\chi^{2}}\left[(1-z)\left(u\left(x_{i}, t_{j}\right)-\chi u^{\prime}\left(x_{i}, t_{j}\right)+\frac{1}{2} \chi^{2} u^{\prime \prime}\left(x_{i}, t_{j}\right)-\frac{1}{6} \chi^{3} u^{3}\left(x_{i}, t_{j}\right)+\frac{1}{24} \chi^{4} u^{4}\left(x_{i}, t_{j}\right)-\frac{1}{120} \chi^{5} u^{5}\left(x_{i}, t_{j}\right)+\cdots\right)\right. \\
& +(3 z-2) u\left(x_{i}, t_{j}\right)+(1-3 z)\left(u\left(x_{i}, t_{j}\right)-\chi u^{\prime}\left(x_{i}, t_{j}\right)+\frac{1}{2} \chi^{2} u^{\prime \prime}\left(x_{i}, t_{j}\right)-\frac{1}{6} \chi^{3} u^{3}\left(x_{i}, t_{j}\right)\right. \\
& \left.+\frac{1}{24} \chi^{4} u^{4}\left(x_{i}, t_{j}\right)-\frac{1}{120} \chi^{5} u^{5}\left(x_{i}, t_{j}\right)+\cdots\right)+z\left(u\left(x_{i}, t_{j}\right)-\chi u^{\prime}\left(x_{i}, t_{j}\right)+\frac{1}{2}(2 \chi)^{2} u^{\prime \prime}\left(x_{i}, t_{j}\right)-\frac{1}{6}(2 \chi)^{3} u^{3}\left(x_{i}, t_{j}\right)\right. \\
& \left.\left.+\frac{1}{24}(2 \chi)^{4} u^{4}\left(x_{i}, t_{j}\right)-\frac{1}{120}(2 \chi)^{5} u^{5}\left(x_{i}, t_{j}\right)+\cdots\right)\right] \\
& -\left(u^{\prime \prime}\left(x_{i}, t_{j}\right)+z \chi u^{3}\left(x_{i}, t_{j}\right)+\frac{1}{2}(z \chi)^{2} u^{4}\left(x_{i}, t_{j}\right)+\frac{1}{6}(z \chi)^{3} u^{5}\left(x_{i}, t_{j}\right)+\cdots\right) \\
= & \left(\frac{3}{4}-\frac{1}{12} z-\frac{z^{2}}{2}\right) \chi^{2} u^{4}\left(x_{i}, t_{j}\right)+\left(\frac{1}{4}+\frac{1}{30} z+\frac{z^{3}}{6}\right) \chi^{3} u^{5}\left(x_{i}, t_{j}\right)+O \chi^{4},
\end{aligned}
$$

we can found that the operator $D_{\chi, z}^{(2)} U_{i}^{j}$ in general is secondorder accurate, and if $z=((3-\sqrt[\sqrt{15}]{)}) / 6)$, it is third-order accurate.

These schemes can be used to create the following difference operator $\Lambda_{\chi, z}$ :

$$
\begin{aligned}
\Lambda_{\chi, z} U_{i}^{j}= & -\varepsilon D_{\chi, z}^{(2)} U_{i}^{j}-a\left(x_{i+z}, t_{j}\right) D_{\chi, z}^{(1)} U_{i}^{j} \\
& +c\left(x_{i+z}, t_{j}\right) D_{\chi, z}^{(0)} U_{i}^{j}, \quad i=1, \ldots, \frac{N}{2}-2,
\end{aligned}
$$


where $z=((3-\sqrt{15}) / 6)$ and $\chi=h$. The operator $\Lambda_{\chi, z}$ is only used as part of the discretization on the Shishkin grid because the Shishkin grid is not uniform in the entire computational domain. More specifically, the difference operator $\Lambda_{\chi, z}$ cannot be applied at $x_{(N / 2)-1}$ and $x_{N-1}$.

Remark 1. Because scheme $\Lambda_{\chi, z}$ has point $\left(x_{i+2}, t_{j}\right)$ in it, the Shishkin grid used is divided into two intervals, $\left[0, x_{N / 2}\right]$ and $\left[x_{(N / 2)+1}, x_{N}\right]$. Since the step size is different and the point $x_{i+2}$ spans two intervals, the difference operator $\Lambda_{\chi, z}$ cannot be applied at either $x_{(N / 2)-1}$ or $x_{N-1}$.

Now, we introduce the difference operator as follows:

$$
\begin{aligned}
\tilde{\Lambda}_{\chi, z} U_{i}^{j}= & -\varepsilon D^{\prime \prime} U_{i}^{j}+a\left(x_{i+z}, t_{j}\right) D_{\chi, z}^{(1)} U_{i}^{j} \\
& +c\left(x_{i+z}, t_{j}\right) D_{\chi, z}^{(0)} U_{i}^{j}, \quad i=\frac{N}{2}+1, \ldots, N-1,
\end{aligned}
$$

with

$$
D^{\prime \prime} U_{i}^{j}=\frac{2}{h_{i}^{j}+h_{i+1}^{j}}\left(\frac{U_{i+1}^{j}-U_{i}^{j}}{h_{i+1}^{j}}-\frac{U_{i}^{j}-U_{i-1}^{j}}{h_{i}^{j}}\right),
$$

where $z=(1 / \sqrt{3})$ and $\chi=H$, and it is also third-order accurate.

Then, we give the scheme at point $x_{N / 2}$ by means of oneside difference schemes as follows:

$$
\begin{aligned}
\widehat{\Lambda}_{H, 1-z} U_{N / 2}^{j}= & -\varepsilon \widehat{D}^{\prime \prime} U_{N / 2}^{j}+a\left(x_{(N / 2)+1-z}, t_{j}\right) D_{H, 1-z}^{(1)} U_{N / 2}^{j} \\
& +c\left(x_{(N / 2)+1-z}, t_{j}\right) \bar{D}^{(0)} U_{N / 2}^{j},
\end{aligned}
$$

with

$$
\bar{D}^{(0)} U_{N / 2}^{j}=\left(\frac{1}{6}+\frac{z}{2}\right) U_{N / 2}^{j}+\frac{2}{3} U_{(N / 2)+1}^{j}+\left(\frac{1}{6}-\frac{z}{2}\right) U_{(N / 2)+2}^{j},
$$

and

$$
\widehat{D}^{\prime \prime} U_{N / 2}^{j}=\frac{1}{H^{2}}\left(U_{N / 2}^{j}-2 U_{(N / 2)+1}^{j}+U_{(N / 2)+2}^{j}\right),
$$

where $z=1-(1 / \sqrt{3})$ and $\chi=H$, and both $\bar{D}^{(0)} U_{N / 2}^{j}$ and $\widehat{D}^{\prime \prime} U_{N / 2}^{j}$ have third-order accuracy in space.

In addition, about the $t$-direction, the discretization of $u_{t}\left(x_{i}, t_{j}\right)$ by the backward Euler scheme is defined by

$$
D_{t}^{-} U_{i}^{j}=\frac{U_{i}^{j}-U_{i}^{j-1}}{\Delta t} \approx u_{t}\left(x_{i}, t_{j}\right)
$$

with the time step $\Delta t$, and it is first-order accurate with respect to the temporal variable $t$.

Finally, we combine $D_{t}^{-} U_{i}^{j}$ with three difference operators $\Lambda_{\chi, z} U_{i}^{j}, \widetilde{\Lambda}_{\chi, z} U_{i}^{j}$, and $\widehat{\Lambda}_{\chi, z} U_{i}^{j}$ at different points, respectively, and finally propose the following numerical scheme:

$$
\begin{cases}\Lambda_{h, z} U_{i}^{j}+D_{t}^{-} U_{i}^{j}=f_{i+z}^{j}\left(z=\frac{3-\sqrt{15}}{6}\right), & \text { for } 1 \leq i \leq \frac{N}{2}-2, \\ \tilde{\Lambda}_{h, z} U_{i}^{j}+D_{t}^{-} U_{i}^{j}=f_{i+z}^{j}\left(z=\frac{1}{\sqrt{3}}\right), & \text { for } i=\frac{N}{2}-1, \\ \widehat{\Lambda}_{H, z} U_{i}^{j}+D_{t}^{-} U_{i}^{j}=f_{i+z}^{j}\left(z=1-\frac{1}{\sqrt{3}}\right), & \text { for } i=\frac{N}{2}, \\ \tilde{\Lambda}_{H, z} U_{i}^{j}+D_{t}^{-} U_{i}^{j}=f_{i+z}^{j}\left(z=\frac{1}{\sqrt{3}}\right), & \text { for } \frac{N}{2}+1 \leq i \leq N-1,\end{cases}
$$

with $j=1, \ldots, M$.

\section{Linear Problem}

The corresponding difference schemes of $u_{x x}, u_{x}$, and $u$ at point $\left(x_{i+z}, t_{j}\right)$ and $u_{t}$ at point $\left(x_{i}, t_{j}\right)$ are substituted into equation (1). When combined with scheme (32), the following linear equations (33) are obtained:

$$
r_{1} U_{i-1}^{j}+r_{2} U_{i}^{j}+r_{3} U_{i+1}^{j}+r_{4} U_{i+2}^{j}=g^{j},
$$

where $U_{i}^{j}$ is the approximation of $u\left(x_{i}, t_{j}\right)$ and $r_{1}, r_{2}, r_{3}, r_{4}$, and $g^{j}$ are defined as follows: if $1 \leq i \leq(N / 2)-2$, 


$$
\left\{\begin{array}{l}
r_{1}=\frac{-\varepsilon(1-z) \Delta t}{h^{2}}-\frac{\Delta t b\left(x_{i+z}, t_{j}\right)\left(-3 z^{2}+6 z-2\right)}{6 h}+\frac{1}{2} \Delta t z(z-1)+\frac{1}{2} z(z-1), \\
r_{2}=\frac{-\varepsilon \Delta t(3 z-2)}{h^{2}}-\frac{\Delta t b\left(x_{i+z}, t_{j}\right)\left(3 q^{2}-4 z-1\right)}{2 h}+\Delta t\left(1-z^{2}\right)+z(z+1), \\
r_{3}=\frac{-\varepsilon \Delta t(1-3 z)}{h^{2}}-\frac{\Delta t b\left(x_{i+z}, t_{j}\right)\left(-3 z^{2}+2 z+2\right)}{2 h}+\frac{1}{2} \Delta t z(z+1)+\frac{1}{2} z(z+1), \\
r_{4}=\frac{-\varepsilon \Delta t z}{h^{2}}-\frac{\Delta t b\left(x_{i+z}, t_{j}\right)\left(3 z^{2}-1\right)}{6 h} \\
g^{j}=\Delta t f\left(x_{i+z}, t_{j}\right)+D_{h, z}^{(0)} U_{i}^{j-1},
\end{array}\right.
$$

where $\Delta t$ is the time steps, $h$ is the space steps defined by (7), $z=((3-\sqrt{15}) / 6)$, and $D_{h, z}^{(0)} U_{i}^{j-1}$ is defined by (11). If $i=(N / 2)-1$,

$$
\left\{\begin{array}{l}
r_{1}=\frac{-\varepsilon \Delta t}{h^{2}}-\frac{\Delta t b\left(x_{i+z}, t_{j}\right)\left(-3 z^{2}+6 z-2\right)}{6 h}+\frac{1}{2} \Delta t z(z-1)+\frac{1}{2} z(z-1), \\
r_{2}=\frac{\varepsilon \Delta t}{h^{2}}+\frac{\varepsilon \Delta t}{h^{2}}-\frac{\Delta t b\left(x_{i+z}, t_{j}\right)\left(3 z^{2}-4 z-1\right)}{2 h}+\Delta t\left(1-z^{2}\right)+\left(1-z^{2}\right), \\
r_{3}=\frac{-\varepsilon \Delta t}{h^{2}}-\frac{\Delta t b\left(x_{i+z}, t_{j}\right)\left(-3 z^{2}+2 z+2\right)}{2 h}+\frac{1}{2} \Delta t z(z+1)+\frac{1}{2} z(z-1), \\
r_{4}=0,
\end{array}\right.
$$


where $\Delta t$ is the time steps, $h$ is the space steps defined by (7), $z=(1 / \sqrt{3})$, and $D_{h, z}^{(0)} U_{i}^{j-1}$ is defined by (11). If $i=(N / 2)$,

$$
\left\{\begin{array}{l}
r_{1}=0, \\
r_{2}=\frac{-\varepsilon \Delta t}{H^{2}}-\frac{\Delta t b\left(x_{i+z}, t_{j}\right)\left(3 z^{2}-2 z-2\right)}{2 H}+\frac{1}{2} \Delta t\left(\frac{1}{3}+z\right)+\frac{1}{2}\left(\frac{1}{3}+z\right) \\
r_{3}=\frac{-\varepsilon \Delta t z}{H^{2}}-\frac{\Delta t b\left(x_{i+z}, t_{j}\right)\left(3 z^{2}-1\right)}{6 H}+\frac{2}{3} \Delta t+\frac{2}{3}, \\
r_{4}=\frac{-\varepsilon \Delta t}{H^{2}}-\frac{\Delta t b\left(x_{i+z}, t_{j}\right)\left(3 z^{2}-6 z+2\right)}{6 H}+\frac{1}{2} \Delta t\left(\frac{1}{3}-z\right)+\frac{1}{2}\left(\frac{1}{3}-z\right) \\
g^{j}=\Delta t f\left(x_{i+1-z}, t_{j}\right)+\bar{D}^{(0)} U_{N / 2}^{j-1},
\end{array}\right.
$$

where $\Delta t$ is the time steps, $H$ is the space steps defined by (7), $z=1-(1 / \sqrt{3})$, and $\bar{D}^{(0)} U_{N / 2}^{j-1}$ is defined by (11). If $(N / 2)+1 \leq i \leq N-1$,

$$
\left\{\begin{array}{l}
r_{1}=\frac{-\varepsilon \Delta t}{H^{2}}-\frac{\Delta t b\left(x_{i+z}, t_{j}\right)\left(-3 z^{2}+6 z-2\right)}{6 H}+\frac{1}{2} \Delta t z(z-1)+\frac{1}{2} z(z-1), \\
r_{2}=\frac{\varepsilon \Delta t}{H^{2}}+\frac{\varepsilon \Delta t}{H^{2}}-\frac{\Delta t b\left(x_{i+z}, t_{j}\right)\left(3 z^{2}-4 z-1\right)}{2 H}+\Delta t\left(1-z^{2}\right)+\left(1-z^{2}\right), \\
r_{3}=\frac{-\varepsilon \Delta t}{H^{2}}-\frac{\Delta t b\left(x_{i+z}, t_{j}\right)\left(-3 z^{2}+2 z+2\right)}{2 H}+\frac{1}{2} \Delta t z(z+1)+\frac{1}{2} z(z+1), \\
r_{4}=0,
\end{array}\right.
$$


where $\Delta t$ is the time steps, $H$ is the space steps defined by (7), $z=(1 / \sqrt{3})$, and $D_{H, z}^{(0)} U_{i}^{j-1}$ is defined by (11).

Finally, the linear system for numerical scheme (32) is obtained, that is,

$$
\widehat{A} x=g .
$$

Here, the coefficient matrix $\widehat{A}$ is defined by

$$
\begin{aligned}
& \text { row } \\
& 1 \\
& 2
\end{aligned}
$$

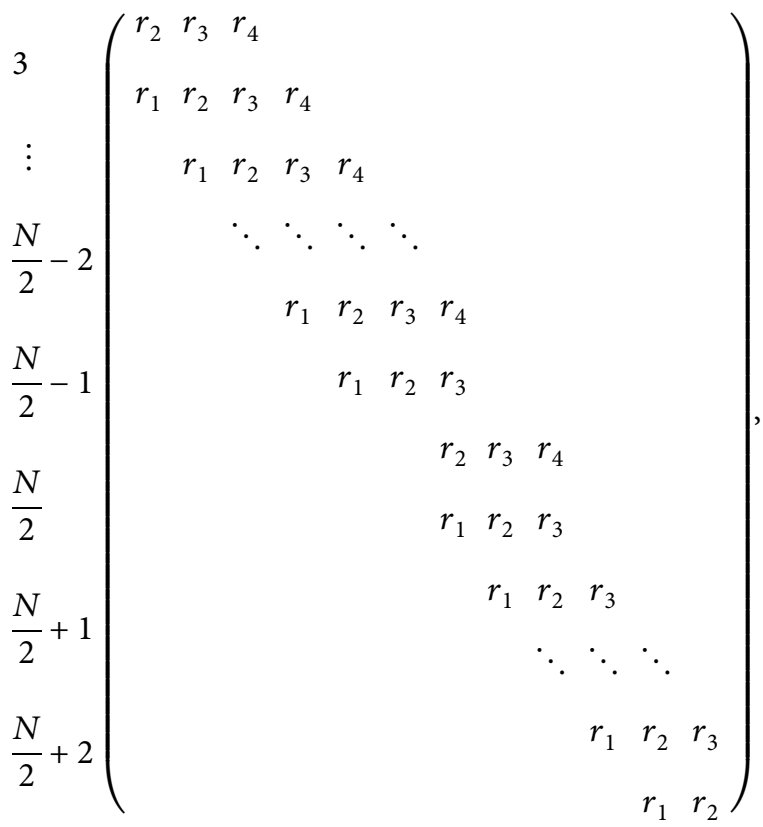

$$
\begin{aligned}
& N-2 \\
& N-1
\end{aligned}
$$

where each unwritten element is 0 . The unknown term $x$ is defined by $\left(x_{0}, x_{1}, x_{2}, \ldots, x_{N-1}, x_{N}\right)^{T}$ with $x_{0}=0$ and $x_{N}=1$, and the right end term $g$ is defined by $\left(g_{0}, g_{1}, g_{2}, \ldots, g_{N-1}, g_{N}\right)^{T}$.

\section{Preconditioning}

In this section, we analyze the $(N-1) \times(N-1)$ matrix $\widehat{A}$, which corresponds to the first scheme (32) and is acquired in Section 4 . We need to assume (4) and that $N$ is sufficiently large and

$$
N \geq N_{*}
$$

where $N_{*}$ is a positive integer independent of $\varepsilon$. There exist constants $N_{*}$ and $C_{*}$ such that (4) and (40) are satisfied, and the matrix $\widehat{A}$ has the following structure:

$$
\begin{aligned}
& \text { row } \\
& 1 \\
& 2
\end{aligned}
$$

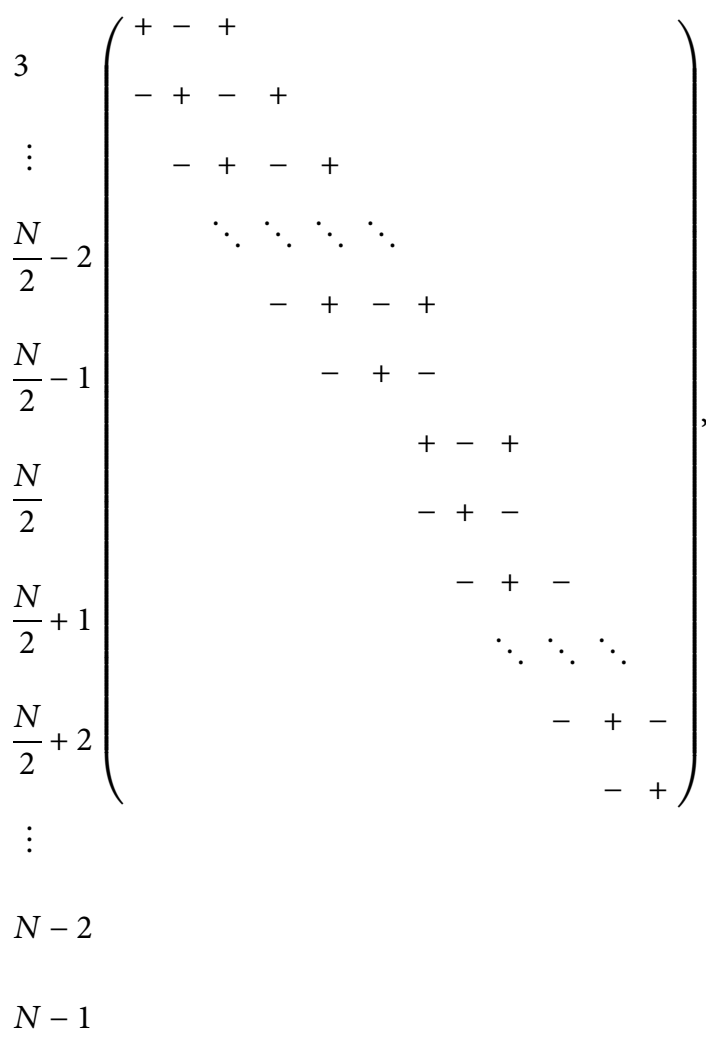

where each unwritten element is 0 .

Multiply its equations $1,2, \ldots,(N / 2)-1$ by $(h / H)$. We do this to achieve consistency uniform in $\varepsilon$, but at the same time, the coefficient matrix gets preconditioned as well (the preconditioning be described in $[18,19])$.

Thus, we take the matrix of the preconditioned system as follows:

$$
\widehat{B}:=\operatorname{diag}\left(m_{1}, m_{2}, \ldots, m_{N-1}\right) \widehat{A}
$$

where

$$
m_{i}= \begin{cases}\frac{h}{H}, & \text { for } i=0,1,2, \ldots, \frac{N}{2}-1, \\ 1, & \text { for } i=\frac{N}{2}, \ldots, N-1 .\end{cases}
$$

\section{Pseudo Code}

In this section, the pseudo code needed to solve problem (1) using numerical scheme (32) in MATLAB will be presented. In general, if mathematical tools are used to solve problem like this, by scheme (32), there are six steps as follows [17]:

(1) Set the uniform mesh for temporal variable ( $M$ is the total number of points in $t$-direction) 
(2) Set the Shishkin mesh for spatial variable ( $N$ is the total number of points in space)

(3) Write down the coefficient matrix $\widehat{A}$ and the right end term $g$ for the linear system as follows:

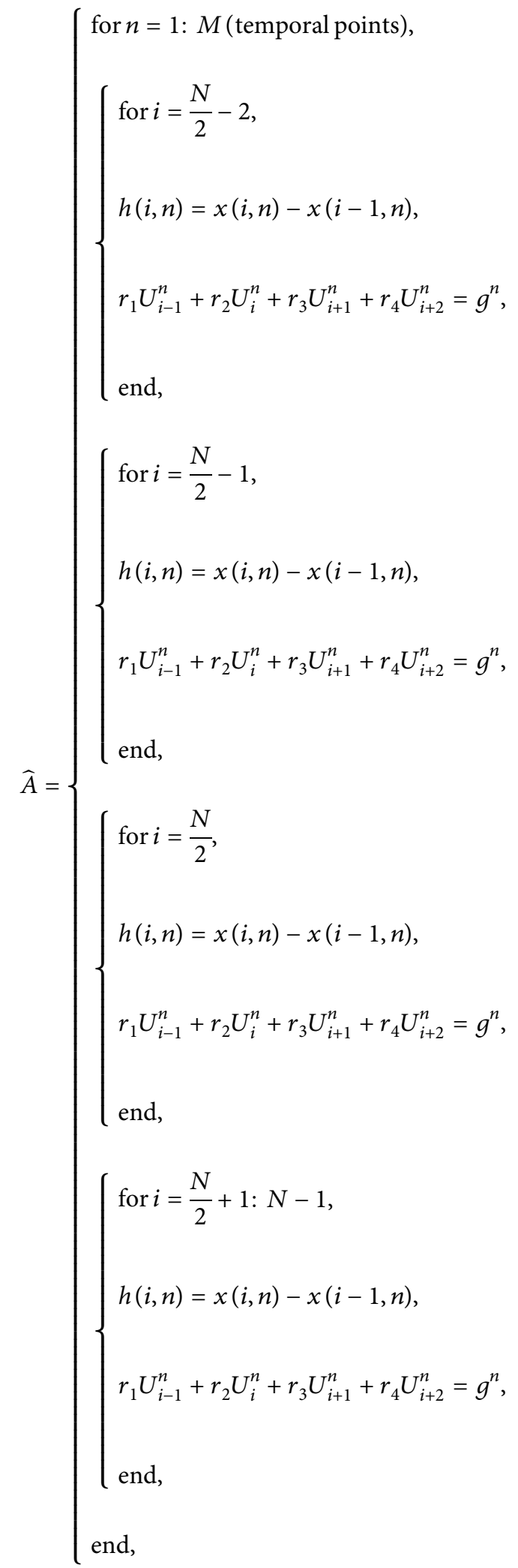

where $U_{i}^{n}$ is the approximation of $u\left(x_{i}, t_{n}\right), h(i, n)$ is the space steps, $r_{1}, r_{2}, r_{3}$, and $r_{4}$ are the elements of the coefficient matrix, and $g^{n}$ is the right end term (it is defined in Section 4)

(4) A new matrix $\widehat{B}$ (from Section 5) is obtained by preprocessing the matrix $\widehat{A}$

(5) The new matrix $\widehat{B}$ and the right end term $g$ are used to solve problem (1)

(6) The maximum pointwise errors and the orders of convergence are calculated

\section{Numerical Experiments}

In this section, we shall present the numerical results obtained by the proposed numerical schemes (32) for the test problem (45) on the piecewise-uniform rectangular mesh $G=\Omega_{x}^{N} \times \Omega_{t}^{M}$. In both cases, we perform the numerical experiments by choosing the constants $\alpha=4$ and $\beta=1$ in (6) and the time step $\Delta t=(1.0 / M)$.

For numerical tests, we consider the following singularly perturbed parabolic problem:

$$
\left\{\begin{array}{l}
-\varepsilon \frac{\partial^{2} u}{\partial x^{2}}-(x+1) \frac{\partial u}{\partial x}+u+\frac{\partial u}{\partial t}=f(x, t), \quad(x, t) \in G, \\
u(0, t)=u(1, t)=0, \quad t \in[0,1], \\
u(x, 0)=0, \quad x \in[0,1],
\end{array}\right.
$$

where $G:=(0,1) \times(0,1]$. We choose the initial data $u(x, 0)=0$ and the exact solution for problem (45) as follows [16]:

$$
u(x, t)=t\left(e^{-(x / \varepsilon)}-e^{x}+\left(e-e^{-(1 / \varepsilon)}\right) x\right) .
$$

As the exact solution of problem (45) is known, we calculate the maximum pointwise error by

$$
E_{\varepsilon}^{N, \Delta t}=\max \left|u_{i, j}-U_{i, j}^{N, \Delta t}\right|,
$$

for each $\varepsilon$, where $u_{i, j}$ and $U_{i, j}^{N, \Delta t}$ denote the exact solution and numerical solution on $\left(x_{i}, t_{j}\right)$, respectively. The convergence order is calculated by the following formula:

$$
R_{\varepsilon}^{N, \Delta t}=\log _{2}\left[\frac{E_{\varepsilon}^{N, \Delta t}}{E_{\varepsilon}^{2 N,(\Delta t / 2)}}\right] .
$$

The maximum pointwise errors $E_{\varepsilon}^{N, \Delta t}$ and the order of convergence $R_{\varepsilon}^{N, \Delta t}$ by using schemes (32) are presented in Table 1 . In table, we can observe the $\varepsilon$-uniform convergence of the numerical scheme. The order of convergence in Table 1 is first-order due to the effect of time error. In order to justify the spatial order of convergence precisely, we take $M=N^{3}$ and the order of convergence is defined by 
TABle 1: Temporal errors and converge orders of scheme (32), $E_{\varepsilon}, R_{\varepsilon}$.

\begin{tabular}{lccccc}
\hline \multicolumn{5}{c}{$N, \Delta t$} \\
\hline$\varepsilon$ & $32,(1 /(32))$ & $64,(1 /(64))$ & $128,(1 /(128))$ & $256,(1 /(256))$ & $512,(1 /(512))$ \\
$10^{-2}$ & 0.01367 & 0.006294 & 0.002864 & 0.001293 & $5.788 e-04$ \\
$10^{-2}$ & 1.12 & 1.14 & 1.15 & 1.15 & - \\
$10^{-4}$ & 0.02067 & 0.010505 & 0.005285 & 0.00265 & 0.001325 \\
$10^{-4}$ & 0.9710 & 0.9910 & 0.9966 & - & $R_{\varepsilon}$ \\
$10^{-6}$ & 0.02075 & 0.01055 & 0.005315 & 0.9989 & 0.001335 \\
$10^{-6}$ & 0.9754 & 0.9898 & 0.9954 & 0.9977 & - \\
$10^{-8}$ & 0.02075 & 0.01055 & 0.005315 & 0.002665 & 0.001335 \\
$10^{-8}$ & 0.9754 & 0.9898 & 0.9954 & 0.9983 & - \\
\hline
\end{tabular}

TABle 2: Spatial errors and converge orders of scheme (32), $E_{\varepsilon}, R_{\varepsilon}$.

\begin{tabular}{lccccc}
\hline \multicolumn{5}{c}{$N, \Delta t$} \\
\hline$\varepsilon$ & $32,\left(1 /(32)^{3}\right)$ & $64,\left(1 /(64)^{3}\right)$ & $128,\left(1 /(128)^{3}\right)$ & $256,\left(1 /(256)^{3}\right)$ & $512,\left(1 /(512)^{3}\right)$ \\
$10^{-2}$ & 0.0088 & 0.0021 & $4.538 e-04$ & $8.644 e-05$ & $1.453 e-05$ \\
$10^{-2}$ & 2.05 & 2.22 & 2.39 & 2.57 & - \\
$10^{-4}$ & 0.0088 & 0.0021 & $4.538 e-04$ & $8.644 e-05$ & $E_{\varepsilon}$ \\
$10^{-4}$ & 2.05 & 2.22 & 2.39 & 2.57 & $-1.453 e-05$ \\
$10^{-6}$ & 0.0089 & 0.0022 & $4.715 e-04$ & $9.397 e-05$ & - \\
$10^{-6}$ & 2.03 & 2.22 & 2.31 & 2.44 & $1.739 e-05$ \\
$10^{-8}$ & 0.0089 & 0.0022 & $4.714 e-04$ & $9.397 e-05$ & - \\
$10^{-8}$ & 2.04 & 2.20 & 2.33 & 2.43 & $1.7393 e-05$ \\
\hline
\end{tabular}

$$
R_{\varepsilon}^{N, \Delta t}=\log _{2}\left[\frac{E_{\varepsilon}^{N, \Delta t}}{E_{\varepsilon}^{2 N,(\Delta t / 8)}}\right]
$$

The numerical results are presented in Table 2, where the spatial convergence order is almost third-order.

\section{Conclusion}

A hybrid scheme is proposed for obtaining a numerical solution to the singularly perturbed parabolic problem. The idea is based on the methods presented in the existing research study [15-19]. It can be seen from the results of numerical experiments, whether in space or in time, the scheme is robust insomuch the error of the numerical solution does not increase when $\varepsilon \longrightarrow 0$. On the contrary, the proposed schemes improve as $\varepsilon$ diminishes, becoming almost third-order accurate with the spatial variable and firstorder accurate with the temporal variable. The numerical results were compared with those from literature $[15-18,20,21]$ which showed that all results reach the expected order of convergence. However, so far it is not possible to construct an arbitrary high-order difference scheme for Shishkin grids, meaning further research is needed. It should be noted that parallelization is not discussed in this article, so the reader is encouraged to refer to additional work $[20,21]$.

\section{Data Availability}

The data used to support the findings of this study are openly available in web of science at https://doi.org/10.1016/j.amc. 2020.125495.

\section{Conflicts of Interest}

The authors declare that they have no conflicts of interest.

\section{Acknowledgments}

This research was partially supported by National Natural Science Foundation of China (11771257 and 11601251).

\section{References}

[1] J. Lorenz, "Stability and monotonicity properties of stiff quasilinear boundary value problems," Review of Research Faculty of Science-University of Novi Sad, vol. 12, pp. 151$175,1982$.

[2] X. Yi, R. Guo, and Y. Qi, "Stabilization of chaotic systems with both uncertainty and disturbance by the UDE-based control method," IEEE Access, vol. 8, no. 1, pp. 62471-62477, 2020.

[3] L. Liu, B. Li, and R. Guo, "Consensus control for networked manipulators with switched parameters and topologies," IEEE Access, vol. 9, pp. 9209-9217, 2021.

[4] T. Hou, Y. Liu, and F. Deng, "Stability for discrete-time uncertain systems with infinite Markov jump and time-delay," Science China Information Sciences, vol. 64, no. 5, pp. 1-11, 2021.

[5] X. Liu, M. Stynes, and J. Zhang, "Supercloseness of edge stabilization on Shishkin rectangular meshes for convectiondiffusion problems with exponential layers," IMA Journal of Numerical Analysis, vol. 38, no. 4, pp. 2105-2122, 2018.

[6] J. Zhang and X. Liu, "Analysis of SDFEM on Shishkin triangular meshes and hybrid meshes for problems with characteristic layers," Journal of Scientific Computing, vol. 68, no. 3, pp. 1299-1316, 2016.

[7] J. Zhang and X. Liu, "Supercloseness of the SDFEM on Shishkin triangular meshes for problems with exponential 
layers," Advances in Computational Mathematics, vol. 43, no. 4, pp. 759-775, 2017.

[8] J. Zhang and X. Liu, "Optimal order of uniform convergence for finite element method on Bakhvalov-type meshes," Journal of Scientific Computing, vol. 85, no. 1, p. 2, 2020.

[9] J. Zhang and X. Liu, "Supercloseness of linear finite element method on Bakhvalov-type meshes for singularly perturbed convection-diffusion equation in 1D," Applied Mathematics Letters, vol. 111, Article ID 106624, 2021.

[10] M. Yang, "Higher-order finite volume element methods based on Barlow points for one-dimensional elliptic and parabolic problems," Numerical Methods for Partial Differential Equations, vol. 31, no. 4, pp. 977-994, 2015.

[11] J. Zhang and Y. Lv, "High-order finite element method on a Bakhvalov-type mesh for a singularly perturbed convectiondiffusion problem with two parameters," Applied Mathematics and Computation, vol. 397, Article ID 125953, 2021.

[12] J. Zhang and M. Stynes, "Supercloseness of continuous interior penalty method for convection-diffusion problems with characteristic layers," Computer Methods in Applied Mechanics and Engineering, vol. 319, pp. 549-566, 2017.

[13] M. Stynes and E. O'Riordan, "A uniformly convergent Galerkin method on a Shishkin mesh for a convection-diffusion problem," Journal of Mathematical Analysis and Applications, vol. 214, no. 1, pp. 36-54, 1997.

[14] H.-G. Roos, M. Stynes, and L. Tobiska, "Robust numerical methods for singularly perturbed differential equations," Springer Series in Computational Mathematics, SpringerVerlag, vol. 24, Springer-Verlag, Berlin, Germany, 2nd edition, 2008.

[15] R. Vulanović, "Higher order monotone schemes for a nonlinear singular perturbation problem," ZAMM: Zeitschrift für Angewandte Mathematik und Mechanik, vol. 68, no. 5, 1988.

[16] R. Vulanović and T. A. Nhan, "Robust hybrid schemes of higher order for singularly perturbed convection-diffusion problems," Journal of Computational and Applied Mathematics, vol. 386, Article ID 125495, 2020.

[17] S. Gowrisankar and S. Natesan, "An efficient robust numerical method for singularly perturbed Burgers' equation," Applied Mathematics and Computation, vol. 346, pp. 385-394, 2019.

[18] A. Das and S. Natesan, "Uniformly convergent hybrid numerical scheme for singularly perturbed delay parabolic convection-diffusion problems on Shishkin mesh," Applied Mathematics and Computation, vol. 271, pp. 168-186, 2015.

[19] H.-G. Roos, "A note on the conditioning of upwind schemes on Shishkin meshes," IMA Journal of Numerical Analysis, vol. 16, no. 4, pp. 529-538, 1996.

[20] J. Vigo-Aguiar and S. Natesan, "A parallel boundary value technique for singularly perturbed two-point boundary value problems," The Journal of Supercomputing, vol. 27, no. 2, pp. 195-206, 2004.

[21] S. Natesan, J. Vigo-Aguiar, and N. Ramanujam, "A numerical algorithm for singular perturbation problems exhibiting weak boundary layers," Applied Mathematics and Computation, vol. 45, no. 1-3, pp. 469-479, 2003. 